Para enlazar con este artículo / To link to this article:

http://dx.doi.org/10.14198/fem.2019.33.01

Para citar este artículo / To cite this article:

Ripio Rodríguez, M. Vanesa. "Otro juego de herramientas: matriz de dominación y resistencia simbólica». En Feminismo/s, 33 (junio 2019): 21-34. Dosier monográfico: Diálogos entre la democracia participativa y la interseccionalidad. Construyendo marcos para la justicia social, coords. Patricia Martínez-García y Jone Martínez-Palacios, DOI: 10.14198/fem.2019.33.01

\title{
OTRO JUEGO DE HERRAMIENTAS: MATRIZ DE DOMINACIÓN Y RESISTENCIA SIMBÓLICA
}

\author{
ANOTHER SET OF TOOLS: \\ MATRIX OF DOMINATION AND SYMBOLIC RESISTANCE
}

\author{
M. Vanesa RIPIO RODRÍGUEZ \\ Universidad Complutense de Madrid \\ mariripi@ucm.es \\ https://orcid.org/0000-0001-9110-4611
}

\section{Resumen}

El objetivo de esta indagación es ejemplificar e insistir en la necesidad de arriesgar y (re)elaborar en lo posible, desde un contexto situado, herramientas conceptuales que hagan posible replantear experimentalmente ciertas cuestiones teóricas y prácticas. Estos experimentos buscan desarrollar una resistencia simbólica creativa en todos los ámbitos.

Haciendo una breve genealogía de la matriz de dominación de Patricia Hill Collins, el artículo examina someramente la manera en la que autores como la propia Collins, Pierre Bourdieu, Ludwig Wittgenstein y Virginia Woolf elaboran su instrumental teórico. Con este análisis, buscamos hallar elementos comunes que ayuden a crear nuevas herramientas de investigación. Estas herramientas deberían reconocer y desmantelar la visión del mundo dominante para producir profundas transformaciones en la realidad social que vivimos e investigamos.

Palabras clave: habitus, matriz de dominación, juego, resistencia, experimento. 


\begin{abstract}
The objective of this inquiry is to insist and illustrate with examples the need of risking and rework as far as possible, within a situated context, a set of conceptual tools. These tools should make possible to set out again certain theoretical and practical questions on an experimental basis. These experiments seek to develop a creative and symbolic resistance in all fields.

Creating a brief genealogy of Patricia Hill Collins' concept of matrix of domination, it summary examines the way in which Ludwig Wittgenstein, Pierre Bourdieu, Virginia Woolf and Collins herself prepare their set of speculative instruments. With this analysis, we hope to find certain common elements that help to create new tools of investigation. Those tools should recognize and dismantle the dominant worldview in order to cause deep changes in our reality.
\end{abstract}

Keywords: habitus, matrix of domination, game, resistance, experiment.

Las herramientas del amo no desmantelarán la casa del amo.

(Audre Lorde, 1979)

\title{
1. UNA CAJA DE HERRAMIENTAS
}

Partimos del constructo matriz de dominación, elaborado por Patricia Hill Collins, como instrumento de análisis de la teoría (y la práctica) interseccional, y en particular, de su especificación como «una estructura estructurante» ${ }^{1}$ (Collins, Difference 34). Esta aclaración hace posible explorar su relación con la noción bourdiana de habitus que es definido como «un sistema de disposiciones duraderas y transferibles, estructuras estructuradas dispuestas a funcionar como estructuras estructurantes, es decir, como principios generadores y organizadores de prácticas y de representaciones» (Bourdieu, Sentido Práctico 86).

Dice Bourdieu que el habitus forma, junto al resto de nociones usadas por él para pensar la práctica (campo, capital, estrategia...), una caja de herramientas. La analogía está tomada de las Investigaciones Filosóficas de Ludwig Wittgenstein:

1. La traducción de los textos de Patricia Hill Collins es nuestra.

Feminismo/s 33, junio 2019, pp. 21-34 
Piensa en las herramientas de una caja de herramientas: hay un martillo, unas tenazas, una sierra, un destornillador, una regla, un tarro de cola, cola, clavos y tornillos. Tan diversas como las funciones de estos objetos, tan diversas son las funciones de las palabras (y hay semejanzas aquí y allí). (11)

$\mathrm{Al}$ acudir a la analogía wittgensteiniana, Bourdieu señala que las funciones de esas nociones (habitus, campo, capital...etc.) son, asimismo, tan diversas como las funciones de esos objetos en la caja. Pero, sobre todo, que los dos términos que se comparan, por ejemplo, la llave inglesa y el habitus, sólo tienen sentido cuando se las pone a funcionar sobre una realidad dada. Cada una de ellos sirve para realizar algún trabajo de indagación práctica.

Como las otras, las herramientas conceptuales han sido creadas por el/la teórico/a social para llevar a cabo ciertas acciones, también técnicas, dirigidas a conocer los objetos (y sujetos) del mundo social. Usando esos tecnicismos, manipulamos aquellos objetos, los sometemos a ciertos experimentos (o experiencias), descomponemos sus partes, dibujamos sus elementos y su estructura para conocerlos. Pero también, y esta es la novedad que introducen las nociones de habitus y matriz de dominación en cuanto «estructuras estructurantes», se vuelve posible comprender y acceder al modo de producción de realidad de esos sujetos, es decir, su capacidad estructurante del mundo mientras siguen actuando.

\section{LOS DOS CAJONES DE LA CAJA}

La doble definición del habitus como «estructura estructurada»y «estructura estructurante» supone una compleja y misteriosa relación recursiva entre ambos términos, que Loïc Wacquant presenta como una «doble vida de las estructuras en las cosas y en los cuerpos» (Bourdieu y Wacquant 30). El estudio de la vida práctica implica echar mano de lo que el mismo autor se representa como unas «lentes de doble enfoque».

\subsection{Lentes de doble enfoque}

Esta bipolaridad visual que, al mismo tiempo, une y divide la tarea teórica, tiene que ver con la doble distribución del instrumental de esa caja de herramientas: a la izquierda, encontramos los instrumentos de la Física social (campo y capital) que investigan los objetos del mundo social, para extraer

Feminismo/s 33, junio 2019, pp. 21-34 
de ellos sus elementos y su estructura, incluidos los sujetos en la medida que también pueden ser considerados en cuanto objetos y ocupan un espacio y un tiempo; a la derecha, están los de la Fenomenología social (principalmente, el habitus), que estudia los sujetos en tanto sujetos estructurantes del mundo y, por eso, la práctica en cuanto práctica.

\subsection{El mapa de la Física social}

En el laboratorio de la Física social, se aíslan las estructuras objetivadas, objetos estructurados, cosas estructuradas (también los cuerpos de los sujetos, como puntos en el campo), es decir, las «estructuras estructuradas». Bourdieu describe el espacio social a través de una representación abstracta, un mapa, «un conjunto de puntos a partir de los cuales los agentes (también el sociólogo y sus lectores y lectoras) dirigen sus miradas al mundo social» (Bourdieu, Distinción 169). Para llevar a cabo un análisis de las posiciones relativas en ese espacio social, representa el espacio práctico de la existencia cotidiana como un espacio geométrico topológico (campo). En ese campo, una cosa o un agente tiene lugar (topos) como una posición en relación a otras posiciones.

El análisis estructural exige que se analicen los principios fundamentales que son pertinentes para la construcción del campo de que se trate. Por ejemplo, en la sociedad que Bourdieu estudia en La Distinción, esos principios de construcción son el volumen o cantidad de poderes o de fuerzas sociales (especies de capital) en juego y la estructura o composición de las mismas. Una especie de capital es aquello que, poseído por un agente o grupo de agentes, le permite «disponer de un poder, una influencia y, por tanto, existir en el campo en consideración, en lugar de ser considerada/o una cifra desdeñable» (Bourdieu y Wacquant 136). El campo de La Distinción mapea la distribución de esos poderes sociales (principalmente de capital económico y capital cultural) en la Francia de la década de los 70, es decir, el estado de las relaciones de fuerza entre agentes y grupos de agentes, resultado de unas condiciones materiales de existencia en un momento del tiempo. Estas relaciones de fuerza que capta la Física social van a condicionar objetivamente el trabajo de la Fenomenología. 


\subsection{El sentido práctico de la Fenomenología social}

Sobre el mapa de relaciones de fuerza de la Física, Bourdieu sobrepone otro mapa, punto por punto, que «transforma la distribución de capital, resultado global de la distribución de fuerzas [...] en distribución de capital simbólico» (Bourdieu, Distinción 172). El capital simbólico no es una especie diferente de capital sino un estado de esos capitales, no mensurable en términos físicos, si bien depende objetivamente del capital físicamente medido. El estado simbólico del capital solo es captable por las estructuras subjetivas de las que se ocupa la Fenomenología, es decir, por unos «sistemas de disposiciones corporales duraderas y transferibles» que Bourdieu denomina habitus.

El habitus es sentido práctico, capacidad de orientación de los agentes en el mundo social, capaz de encontrar sentido al estado de relaciones de fuerzas dado (campo) que le condiciona. La misteriosa relación es doble y recursiva, el campo (y los poderes sociales pertinentes o capitales) condicionan unos habitus que transforman aquellas relaciones de fuerza en relaciones de sentido. «Las divisiones sociales se convierten en principios de visión y división que organizan la visión del mundo social» (Bourdieu, Distinción 482). Es decir, la experiencia de los límites objetivos se anticipa prácticamente, cuando se convierte en sentido de los límites. Los esquemas de percepción, pensamiento y acción de los habitus engendran pensamientos, percepciones y acciones «con toda libertad controlada [...] siempre tienen como límite las condiciones histórica y socialmente situadas de su producción» (Bourdieu, Sentido práctico 90).

\subsection{Violencia simbólica}

Este otro mapa desplegado desde el anterior, condicionado por el primero pero no reducible a él, representa el espacio práctico de la vida cotidiana en términos de unas relaciones que discurren por «los caminos puramente simbólicos de la comunicación y del conocimiento o, más exactamente, del desconocimiento, del reconocimiento o, en último término, del sentimiento» (Bourdieu, Dominación 12). La Fenomenología social elabora como herramienta fundamental estas estructuras subjetivadas, encarnadas, somatizadas que son los habitus, es decir, estructuras orientadas por ese mapa. Mapa que ellas mismas no dejan de (re)dibujar, de (re)estructurar, orientándose 
y orientando las cosas del mundo y a los sujetos que lo habitan. Es, en este sentido, que pueden ser definidas como «estructuras estructurantes» del mundo social.

Pero esa labor simbólica estructurante está condicionada por un estado de relaciones de fuerza previo, es decir, por el sometimiento a los poderes sociales (capitales) dados en un campo. Esta situación de libertad controlada, de encontrar sentido a los límites es continuamente (re)conocida de un modo no consciente por la labor (re)estructurante de los habitus. La noción de violencia simbólica hace posible (re)introducir el papel del cuerpo, de las emociones y los sentimientos en el proceso de (re)conocimiento/(des)conocimiento del mundo social. La (re)estructuración del mundo, su (re)producción por parte de los habitus y, por ello, la colaboración (el colaboracionismo) con la visión del mundo dominante, depende del ejercicio y del padecimiento de esa violencia, «violencia amortiguada, insensible e invisible para sus propias víctimas» (Bourdieu, Dominación 11).

La violencia simbólica es esa coerción que se instituye por mediación de esa adhesión que el dominado no puede evitar otorgar al dominante (y, por lo tanto, a la dominación) cuando solo dispone, para pensarlo y pensarse o, mejor aún, para pensar su relación con él, de instrumentos de conocimiento que comparte con él y que al no ser más que la forma incorporada de la estructura de la relación de dominación, hacen que esta se presente como natural. (Bourdieu, Meditaciones 224-5)

La caja de herramientas bourdiana pone en nuestras manos los medios para comprender cómo se (re)produce el mundo social y los propios agentes como «estructuras estructurantes» del mismo, que lo (re)crean al proporcionarles un sentido. Pero, quizá, lo más interesante de usar esas herramientas de comprensión es poner el instrumental en nuestras manos, con el objetivo de crear uno propio.

\section{EN EL LADO FENOMENOLÓGICO: MATRIZ DE DOMINACIÓN Y VIOLENCIA SIMBÓLICA}

Nuestra historia bourdiana, el manejo sistemático que hacemos del instrumental de Bourdieu para comprender los fenómenos de dominación que suelen (pre)ocuparnos, nos lleva a colocar la matriz de dominación de Patricia Hill Collins en el lado fenomenológico de esa caja de herramientas. Aun 
temiendo que vaya en contra de la propia lógica e intención epistemológica que la autora introduce.

La matriz de dominación es la herramienta que se sitúa y nos sitúa en el centro del Pensamiento Feminista Negro de Collins. El mero uso del término Pensamiento Feminista Negro nos pone sobre aviso: «Se dirige a los debates epistemológicos en curso en relación a las dinámicas de poder que sustentan lo que cuenta como conocimiento» (Collins, Thought 273). Y ello, con la intención de alentar un desplazamiento fundamental del paradigma de cómo pensamos la opresión y, por eso mismo, la resistencia. Ese desplazamiento integra inevitablemente qué entendemos por poder: «...una entidad intangible que circula en el interior de una matriz de dominación particular y con el cual los individuos guardan relaciones diversas» (Collins, Thought 274).

Podemos dar por hecho con Collins y a la Bourdieu, que nuestro pensamiento está violentado simbólicamente, que nos vemos obligadas como mujeres, pero también como negras, como gitanas o mediterráneas, lesbianas, de clase obrera, etc..., a sospechar de la caja de herramientas del amo: varón, blanco, heterosexual, etc. Sin duda, la resistencia epistemológica está en pensar de otra manera, pero también en aliarse con aquellos que lo hacen.

La definición de la matriz de dominación como estructura estructurante nos lleva a encontrar similitudes con alguna de las herramientas bourdianas. Esta genealogía posible revela que la matriz no es el habitus, pero que guarda con él similitudes importantes. La matriz es una herramienta interseccional que incide en un modo de funcionamiento específico de las prácticas de dominación. La interseccionalidad es un paradigma complejo y sutil que «nos recuerda que la dominación no se reduce a un tipo fundamental y que las dominaciones colaboran para producir injusticia» (Collins, Thought 18). La matriz de dominación es un instrumento de análisis de esa teoría (y práctica) interseccional que nos permite entender cómo esas dominaciones están organizadas. Dicho de otro modo, la interseccionalidad enfatiza «la relacionalidad entre opresiones que se intersectan» (Collins, Difference 23), que convergen para generar, en cada contexto social y político, «una 'matriz' característica de una dinámica de poderes intersectados (a distinctive 'matrix' of intersecting power dynamics)» (Collins, Difference 22).

Si bien Collins describe su herramienta como una «organización social global en la que se originan, desarrollan y retienen opresiones que se

Feminismo/s 33, junio 2019, pp. 21-34 
intersectan», al mismo tiempo, señala que dicha organización es siempre históricamente específica y que «encapsula la universalidad de opresiones que se intersectan en diversas realidades locales» (Collins, Thought 228). Con ello, Collins transita la distancia entre la abstracción teórica de la estructura de dominación y el principio práctico de la experiencia colectiva y singular. Este último da cuenta de la confluencia en cada agente de unas dominaciones específicas, singularmente cristalizadas en una matriz determinada: «Las mujeres negras nos encontramos con un conjunto de prácticas sociales que acompañan nuestra historia particular en el seno de una matriz de dominación caracterizada por opresiones que se intersectan» (Collins, Thought 23).

Esa «entidad intangible», que llamamos poder, circula en el interior de una matriz de dominación particular, quedando así articulada, organizada, estructurada en tres dominios:

Cada dominio sirve a un propósito particular. El estructural organiza la opresión, mientras que el disciplinario la gestiona. El dominio hegemónico justifica la opresión y el dominio interpersonal influencia la experiencia vivida de cada día y la conciencia individual resultante. (Collins, Thought 276)

Vista en términos más abstractos, más generales, como organización global de dominación y herramienta de la teoría interseccional, la matriz se estructura en diversos ejes de poder, racial, de clase o de género. Vista al nivel de la experiencia y la resistencia a la opresión de la gente, queda organizada en esos tres niveles o dominios de poder. Collins considera que, considerados colectivamente, estos dominios de poder «proporcionan un conjunto de herramientas conceptuales para la elaboración de respuestas de diagnóstico y estrategia en el seno de una matriz de dominación determinada» (Collins, Thought 23).

Encontramos en Collins, como en Bourdieu, una estructura doble, una «doble vida» de la matriz de dominación y, con ello, nos volvemos a topar con aquella relación misteriosa y recursiva entre la estructura estructurada y la estructura estructurante. 


\section{UNA HERRAMIENTA COMPLEJA DE RESISTENCIA EPISTEMOLÓGICA}

La Interseccionalidad enfatiza la relacionalidad (Collins, Difference 23) entre distintos modos de dominación, que confluyen para generar, en cada contexto social y político, «a distinctive 'matrix' of intersecting power dynamics» (Collins, Difference 22). Este nivel macro, abstracto, que investiga los principios similares en los que descansa la dominación política (Collins, Difference 24), queda así orientado hacia el estudio micro de cada organización matricial precisa. La conjugación recursiva de ambos sentidos en la investigación, favorece la comprensión no sólo de cada retícula de dominaciones en su especificidad, sino, asimismo, de la consecución de acciones de resistencia.

Si bien nos hemos esforzado por situar la matriz como parte del instrumental teórico de la mentada disciplina, es justo aclarar su diversidad funcional, que hace posible diferenciarla y, por ello, reinterpretar parte del uso de las nociones de habitus y violencia simbólica, que señalamos arriba como elementos fundamentales de la Fenomenología.

El conjunto de herramientas conceptuales de Collins dirigidas a la elaboración tanto de diagnósticos como de acciones estratégicas de resistencia, han sido deliberadamente producidas para realizar «el análisis de problemas sociales específicos que afectan a poblaciones específicas» (Collins, Thought 23). La matriz opera a un nivel micro, molecular, para remontarse, después, al nivel de la estructura global. Si la relación de condicionamiento del campo sobre el habitus es primera para Bourdieu, para Collins ocurre precisamente lo contrario. El nivel constructivo o interrelacional es primero y determinante del segundo. En este sentido, podríamos hablar de una revolución copernicana o collinsiana en lo tocante a la relación recursiva y misteriosa que subsiste en ambos casos.

El motivo o más bien la necesidad de esta revolución quedó expuesta al comienzo del presente texto. La rebelión se dirige a los debates epistemológicos en curso en relación a las dinámicas de poder que sustentan lo que cuenta como conocimiento. Como pensadora feminista negra, Collins parte de lo más concreto no en general, sino en relación a la dominación. La matriz de dominación se construye desde unas opresiones colectivas muy precisas. En este caso, la raza, la clase y el género. Con ello, Collins busca 
(re)conceptualizar las relaciones de dominación y resistencia y, para ello, necesita un nuevo instrumento. Pero, aún más, con ello investiga las condiciones de posibilidad de la verdad, con la intención de proporcionar un nuevo paradigma constructivo o constructivista de la misma. Uno basado en la urgencia imperativa de la resistencia: «Si el poder como dominación está organizado y opera vía opresiones que se intersectan, entonces la resistencia debe mostrar una complejidad comparable» (Collins, Thought 203). Esta complejidad resulta del análisis fenomenológico individual y colectivo de la propia experiencia oprimida. Pensar la dominación desde un lugar dominado, violentado simbólicamente, exige acciones de resistencia complejas, que conjuren la adhesión a los instrumentos de conocimiento que una larga tradición de pensamiento ha impuesto como legítimos.

La conciencia de la opresión, es decir, el conocimiento que la matriz de dominación produce como herramienta de la teoría y la práctica interseccional, se privilegia como elemento primero, condición necesaria de la labor estructurante, también para la teórica o el teórico social. Que esa tarea estructurante quede condicionada por la conciencia de la opresión y, por lo tanto, por las posibilidades de resistencia, explica que Collins defina su instrumental desde su poder estructurante, ejerciendo así su derecho y deber de resistencia epistemológica.

\section{EXPERIMENTOS EN EL LABORATORIO}

Hemos partido de una relación de semejanza entre las nociones de matriz de dominación y habitus. El objeto del presente artículo no es sólo trazar esta posible genealogía bourdiana de la matriz, sino indagar mediante ella cómo podemos producir desde la teoría y la práctica herramientas de conocimiento y resistencia situadas, que cuenten con experiencias de opresión precisas.

La adhesión a teorías y conceptos que se piensan neutrales violentan continuamente la tarea teórica. Pero, incluso si las mismas han sido creadas con la intención, como ocurre en los casos mencionados de Wittgenstein, Bourdieu y Collins, de liberarnos de esa neutralidad, estas pueden dominar irreflexivamente nuestra manera de hacer teoría. Si la relación con las autoras y los autores de referencia está mediada por la violencia simbólica, también 
lo está la presión por convertirse en un autor o una autora innovador/a en un campo determinado.

Para escapar del concepto cuando piensa qué es «hablar una lengua», Wittgenstein elabora otra analogía: «Se podría decir que el concepto de 'juego' es un concepto de bordes borrosos» (71). De ahí, la necesidad de hablar de juegos de lenguaje (Sprachspiel), para enfatizar que «el hablar la lengua es parte de una actividad o una forma de vida» (23). Como advierte Jaakko Hintikka (285), «los juegos del lenguaje no son juegos en el lenguaje, sino típicamente son juegos jugados por medio del lenguaje». Las palabras derivan su significado por su papel en tales «actividades complejas».

La matriz de dominación es la herramienta conceptual elaborada por Collins para revolucionar epistemológicamente el paradigma mediante el cual pensamos el poder. La toma de conciencia situada que facilita este instrumento hará posible encontrar respuestas que diagnostiquen la dominación y proporcionen estrategias de resistencia precisas a opresiones específicas.

La tarea revolucionaria en teoría social no deja de situarse en la órbita de eso que Wittgenstein denomina un juego del lenguaje. La escritura del presente artículo forma parte de él.

La caja de herramientas conceptuales es también la caja de un juego jugado por medio del lenguaje. Cuando Bourdieu trata de explicar a sus lectoras y lectores intuitivamente qué es un campo, acude al símil de juego. El campo es como si fuera un juego, uno cuyas reglas no son explícitas. No hay conciencia de estar jugando y mucho menos de estar dominada o dominado por el juego. Es un juego incorporado en disposiciones corporales duraderas y transferibles, en «esquemas mentales y corporales que funcionan a manera de patrones simbólicos para las actividades prácticas - conducta, pensamientos, sentimientos y juicios- de los agentes sociales» (Bourdieu y Wacquant 31).

Podríamos pensar que la elaboración de herramientas conceptuales es un juego explícito con unas reglas de juego dadas, a las que debemos hacer honor si queremos participar en él. Pero quizá eso es sólo resultado del juego implícito, de la estructura estructurada que nos condiciona, somatizando un sentido de los límites del juego teórico. Si, por el contrario, consideramos esa producción de instrumentos desde el sentido de resistencia estructurante, a la manera de Collins, la propia operación de producción de las herramientas se torna revolucionaria. Dicho de otra manera, si se busca la revolución 
epistemológica, esta solo puede alojarse en unas herramientas que generen cambios simbólicos al nivel de aquellos esquemas y, por ende, que replanteen radicalmente el juego mismo de hacer herramientas. Unas herramientas que hagan posible generar cambios en esos esquemas y, por ello, en la forma de vida. Tendríamos entonces en nuestras manos la capacidad estructurante para cambiar no sólo el paradigma de conocimiento del poder, sino el poder como tal.

El modo de saltar por encima de nuestra propia sombra en la teoría es tomar conciencia de esta relación con el conocimiento y, por ello, con nuestras prácticas. Son estas prácticas las que participan en ese mismo proceso de estructuración de la realidad. De ahí, que puedan asimismo formar parte de una (re)estructuración experimental del sentido de la realidad, producido por la estructura estructurante. Y sólo podemos hacer eso de algún modo, sin desmontar la práctica, mientras los sujetos están, o mejor, estamos en funcionamiento, actuando y pensando.

\section{LA TENSIÓN CREATIVA DE LA RESISTENCIA SIMBÓLICA}

Proponemos iluminar esas resistencias desde el hallazgo experimental «no sólo crítico, sino creativo» de la ley, alumbrado por Virginia Woolf en Tres Guineas (199).

En dicho texto, el juego social es criticado en su conjunto, sin enmienda posible. Como grupo dominado, las mujeres no deben participar ni siquiera en aquellas acciones que busquen ideales de libertad y justicia social. Los ideales mismos forman parte de la visión dominante: «La vieja palabra libertad no nos sirve tampoco, debido a que no era libertad en el sentido de permiso lo que querían, al igual que Antígona no quería quebrantar las leyes sino hallar la ley» (Woolf 241). No deben dar conferencias, ni escribir artículos tampoco, de lo contrario estarán asentando las condiciones materiales y simbólicas de su nueva dominación. Sólo la práctica experimental en secreto, en todos los ámbitos de la vida social, logrará hallar una nueva ley, un nuevo juego que hará posible salir de la dominación.

Sin duda hay mucha dificultad y riesgo en este planteamiento. Pero la cuestión no es tanto si es posible producir un juego social paralelo desde cero, cuanto si vamos a crear nuevos aparatos de laboratorio que lo hagan 
pensable. La experimentación a la que se refiere Woolf no está marcada con ninguna pauta, pero sí está situada, contextualizada. Por decirlo con Bourdieu, depende de nuestro lugar del campo porque nace de problemas específicos. Sin embargo, este condicionamiento que sin duda organiza una labor crítica más que necesaria, no lo es todo. Woolf sólo nos pide creatividad, es decir, riesgo experimental para nuestras acciones de resistencia. Pensamos en acciones orientadas hacia el experimento vivencial en contextos de participación teórica y práctica »que resalten la tensión creativa entre lo deseable, lo posible, lo probable y lo práctico» (Collins, Difference 34).

\section{BIBLIOGRAFÍA}

Bourdieu, Pierre. Meditaciones pascalianas. Barcelona: Anagrama, 1999.

—. La Distinción. Madrid: Taurus, 2000.

—. La Dominación masculina. Barcelona: Anagrama, 2003.

-.El sentido práctico. Madrid: Siglo XXI, 2008.

-. Las estrategias de la reproducción social. Buenos Aires: Siglo XXI, 2011.

Bourdieu, Pierre y Loïc Wacquant. Invitación a una sociología reflexiva. Buenos Aires: Siglo XXI, 2008.

Collins, Patricia Hill. Black Feminist Thought: Knowledge, Consciousness and the Politics of Empowerment. New York: Routledge, 2000.

-. "The Difference that Power Makes: Intersectionality and Participatory Democracy». Investigaciones Feministas 8 (1/2017): 19-39.

Dunezat, Xavier. «Sexo, raza, clase y etnografía de los movimientos sociales. Herramientas metodológicas para una perspectiva interseccional». Revista de Investigaciones Feministas 8 (1/2017): 95-114.

Hintikka, Jaakko. Ludwig Wittgenstein. Half-Truth and One-a-Half-Truth. Boston: Kluwer, 1992.

Martínez-Palacios, Jone. «Exclusión, profundización democrática e interseccionalidad». Revista de Investigaciones Feministas 8 (1/2017): 53-71.

-. «¿Qué significa participar? Reflexiones sobre la construcción de las imágenes de la participación». Papers 103 (3/2018): 367-393.

Ripio Rodríguez, Vanesa. «Habitus outsider». Revista de Investigaciones Feministas 6 (2015): 139-152. 
Wacquant, Loïc. «Bourdieu Comes to Town: Pertinence, Principles and Applications». International Journal of Urban and Regional Research 42 (2018): 86-105.

—. «Four Transversal Principles for Putting Bourdieu to Work». Anthropological Theory 1 (2018): 3-17.

Wittgenstein, Ludwig. Investigaciones Filosóficas. Madrid: Trotta, 2017. Woolf, Virginia. Tres Guineas. Barcelona: Lumen, 1999. 